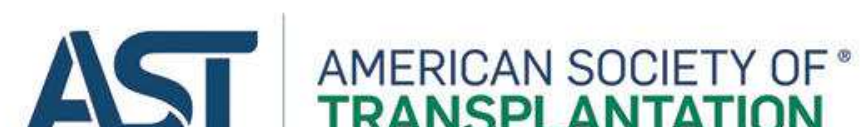 TRANSPLANTATION
}

\section{COMPREHENSIVE}

\section{TRAINEE}

\section{CURRICULUM}

\section{COMPREHENSIVE TRAINEE CURRICULUM}

\section{WATCH 52 ONLINE LESSONS INCLUDING:}

- Adult and Pediatric Liver

- Adult Cardiac and Pulmonary

- Abdominal Transplant Surgery
- Cardiothoracic Transplant Surgery

- Adult and Pediatric Kidney

- Transplant Pharmacy
Solid organ transplantation is a multidisciplinary field, leading to a diverse community of professionals within the AST. As a result, it is often necessary for trainees to have extensive knowledge of all areas of transplantation-not just their specialty.
Check out this brand new resource, meant to supplement the training trainees and fellows receive at their university or hospital.

\$50 members / \$200 non-members 


\title{
Impact of intraoperative cytokine adsorption on outcome of patients undergoing orthotopic heart transplantation-an observational study
}

\author{
Endre Nemeth $^{1}$ (1) | Eniko Kovacs ${ }^{1}$ | Kristof Racz ${ }^{1}$ | Adam Soltesz $^{1}$ | Szabolcs Szigeti ${ }^{1}$ | \\ Nikolett Kiss $^{1}$ | Gergely Csikos ${ }^{1}$ | Kinga B. Koritsanszky ${ }^{1}$ | Viktor Berzsenyi ${ }^{1}$ | \\ Gabor Trembickij $^{1}$ | Szabolcs Fabry ${ }^{1}$ | Zoltan Prohaszka ${ }^{2}$ | Bela Merkely ${ }^{3}$ | Janos Gal ${ }^{1}$
}

${ }^{1}$ Department of Anesthesiology and Intensive Therapy, Semmelweis University, Budapest, Hungary

${ }^{2} 3$ rd Department of Medicine, Research Laboratory, Semmelweis University, Budapest, Hungary

${ }^{3}$ Heart and Vascular Center, Semmelweis University, Budapest, Hungary

Correspondence

Endre Nemeth, Department of Anesthesiology and Intensive Therapy, Semmelweis

University, Budapest, Hungary.

Email: nemeth.endre@med.semmelweis-univ.hu

\begin{abstract}
Aim: The aim of this study was to assess the influence of intraoperative cytokine adsorption on the perioperative vasoplegia, inflammatory response and outcome during orthotopic heart transplantation (OHT).

Methods: Eighty-four OHT patients were separated into the cytokine adsorption (CA)-treated group or controls. Vasopressor demand, inflammatory response described by procalcitonin and C-reactive protein, and postoperative outcome were assessed performing propensity score matching.

Results: In the 16 matched pairs, the median noradrenaline requirement was significantly less in the CA-treated patients than in the controls on the first and second postoperative days $\left(0.14\right.$ vs $0.3 \mu \mathrm{g}^{*} \mathrm{~kg}^{-1 *} \mathrm{~min}^{-1}, P=.039$ and 0.06 vs $0.32 \mu \mathrm{g}^{*} \mathrm{~kg}^{-1 *} \mathrm{~min}^{-1}$, $P=$.047). The inflammatory responses were similar in the two groups. There was a trend toward shorter length of mechanical ventilation and intensive care unit (ICU) stay in the CA-treated group compared to the controls. No difference in adverse events was observed between the two groups. However, the frequency of renal replacement therapy was significantly less in the CA-treated patients than in the controls $(P=.031)$.

Conclusions: Intraoperative CA treatment was associated with reduced vasopressor demand and less frequent renal replacement therapy with a favorable tendency in length of mechanical ventilation and ICU stay. CA treatment was not linked to higher rates of adverse events.
\end{abstract}

KEYWORDS

cardiac surgery, cytosorb, heart transplantation, hemoadsorption, inflammatory response, procalcitonin

\section{1 | INTRODUCTION}

Severe vasoplegia is an important complication of cardiopulmonary bypass (CPB) and cardiac surgery that can develop in $5 \%-44 \%$ of cases. $^{1-3}$ Patients undergoing orthotopic heart transplantation (OHT) are among those of highest risk for perioperative vasoplegic syndrome (VS), with the severity of vasoplegia greater in this group than in nontransplant subjects. ${ }^{2,4,5}$ The perioperative loss of systemic vascular 
resistance with concomitant hypotension and tissue hypoperfusion can adversely influence the length of mechanical ventilation, intensive care unit (ICU) stay, morbidity, and mortality after cardiac surgery as well as $\mathrm{OHT}^{4,5}$ If the vasoplegia is resistant to catecholamines, the mortality rate can be as high as $25 \%{ }^{2}$

The exact pathomechanism of perioperative vasoplegia remains poorly understood. ${ }^{2,4}$ However, it is based on an exaggerated inflammatory response determined by the interaction between the immune system priming and CPB surgery, resulting in excessive release of vasoactive mediators and endothelial injury. ${ }^{2,4,6}$ Patients with advanced stage chronic heart failure are known to have elevated plasma concentrations of pro-inflammatory cytokines and chemokines such as tumor necrosis factor $\alpha$ (TNF- $\alpha$ ), interleukin (IL)-6, and IL-8 correlating with the progression of heart failure. ${ }^{7,8} \mathrm{CPB}$ surgery is also proven to generate systemic inflammatory response syndrome (SIRS) through complement and cytokine activation and ischemia-reperfusion injury. ${ }^{9,10}$ Additionally, the graft tissue induced response of the host immune system can further aggravate these processes in $\mathrm{OHT}^{4}$

The conventional treatment of vasoplegia consists of fluid resuscitation, administration of catecholamine agents, and argininevasopressin or terlipressin. ${ }^{2}$ Nevertheless, using vasoconstrictors in high doses, particularly in noradrenaline-refractory cases, increases the risk of peripheral or mesenteric ischemia, leading to a higher rate of postoperative morbidity and mortality. ${ }^{2,6}$ The use of intravenous methylene blue (MB) as an adjuvant might have the potential to control noradrenaline-refractory VS via the inhibition of nitric oxide synthase and nitric oxide in the pathway of activating soluble guanylate cyclase, a key element in the pathomechanism of VS. ${ }^{2,6,11}$ Despite the significant number of clinical investigations in the field of cardiac and noncardiac surgeries, MB treatment remains controversial in terms of therapeutic effectiveness and in reducing morbidity and mortality. ${ }^{6,11}$

The extracorporeal cytokine hemoadsorption (CytoSorb ${ }^{\mathrm{TM}}$ ) is a novel nonpharmacologic technology, which can effectively remove mid-molecular weight pro- and anti-inflammatory cytokines up to approximately $55 \mathrm{kDa}$ such as TNF- $\alpha$, IL-1 $\beta$, IL-6, and IL-10. ${ }^{12-15}$ Recent animal studies have demonstrated its benefit with hemodynamic stability and outcome in a severe sepsis model. ${ }^{12,14}$ Clinical data of patients with septic shock or post-CPB SIRS have also supported the effectiveness of CytoSorb $^{\text {TM }}$ treatment, showing hemodynamic improvements and reduction of vasoconstrictors. ${ }^{16-19}$

The aim of this observational study was to explore whether preemptive intraoperative use of CytoSorb ${ }^{\text {TM }}$ treatment could influence the severity of perioperative vasoplegia, postoperative inflammatory response, and clinical outcome in patients undergoing OHT.

\section{2 | MATERIALS AND METHODS}

\section{1 | Patients}

This study analyzed prospectively collected clinical data of adult orthotopic heart transplantations performed between January 1, 2015 and December 1, 2016. The study was approved by the Regional and Institutional Committee of Science and Research Ethics, and written informed consent requirement was waived (No: 246/2016). Exclusion criteria were retransplantation and early postoperative death within 72 hours.

\subsection{Anesthetic procedure}

Anesthesia was provided using sufentanyl bolus $0.25 \mu \mathrm{g} / \mathrm{kg}$ intravenously (IV), propofol bolus $1 \mathrm{mg} / \mathrm{kg} \mathrm{IV}$, atracurium bolus $0.3 \mathrm{mg}$ / $\mathrm{kg}$ IV for induction, then a combination of sevoflurane (minimal alveolar concentration: 0.7-1.2), propofol continuous IV infusion (3-5 $\mathrm{mg}^{*} \mathrm{~kg}^{-1 *} \mathrm{~h}^{-1}$ ), and sufentanyl boluses for maintenance of anesthesia including CPB period. Intraoperative monitoring of patients included the standards of cardiac anesthesia including arterial blood pressure, central venous pressure, nasopharyngeal temperature measurements, which was extended with pulmonary artery (PA) pressure monitoring via a PA catheter and continuous transesophageal echocardiography according to the institutional OHT protocol. CPB was provided by roller-pump (SORIN S5 Perfusion System, Sorin Group Deutschland $\mathrm{GmbH}$, Munich, Germany) and a membrane oxygenator (Sorin Inspire P8, Sorin Group Italia Srl, Mirandola, Italy) maintaining nonpulsatile flow rate of $2.4 \mathrm{~L}^{*} \mathrm{~min}^{-1 *} \mathrm{~m}^{-2}$. The CPB prime was composed of $1200 \mathrm{~mL}$ Ringer-lactate, $100 \mathrm{~mL}$ mannitol, and $60 \mathrm{~mL}$ sodium bicarbonate $8.4 \%$. All patients were anesthetized by consultant anesthetists experienced in cardiac anesthesia and OHT.

\section{3 | Perioperative care}

All patients received standardized intra- and postoperative care according to the institutional protocol. Noradrenaline was used as the first line vasopressor, and continuous terlipressin IV infusion $\left(0-2.0 \mu \mathrm{g}^{*} \mathrm{~kg}^{-1 *} \mathrm{~h}^{-1}\right)$ was administered as the second-line vasopressor. Terlipressin infusion was indicated if noradrenaline support exceeded $0.3 \mu \mathrm{g}^{*} \mathrm{~kg}^{-1 *} \mathrm{~min}^{-1}$. The inotrope therapy consisted of the combination of dobutamine (3.0-5.0 $\left.\mu \mathrm{g}^{*} \mathrm{~kg}^{-1 *} \mathrm{~min}^{-1}\right)$ and milrinone $\left(0.35 \mu \mathrm{g}^{*} \mathrm{~kg}^{-1 *} \mathrm{~min}^{-1}\right)$. Patients with confirmed elevated pulmonary vascular resistance (PVR) received inhalational nitric oxide (0-25.0 ppm) during mechanical ventilation (MV) and enteral sildenafil (60 mg/day) as a maintenance therapy in the postoperative period. The major hemodynamic targets were the following: mean arterial blood pressure $(\mathrm{MAP}) \geq 65 \mathrm{~mm} \mathrm{Hg}$, cardiac index $(\mathrm{Cl}) \geq 2.0 \mathrm{~L}^{*} \mathrm{~min}^{-1 *} \mathrm{~m}^{-2}, \mathrm{PVR}<3.0$ Wood unit, and mixed venous oxygen saturation $\left(\mathrm{SvO}_{2}\right) \geq 65 \%$. Intraoperative invasive hemodynamic monitoring was extended for the first 48 hours routinely. Function of the cardiac allograft was checked with echocardiography (transthoracic or transesophageal) daily during the first five post-transplant days and on a weekly basis thereafter.

We applied differential hemostasis management using fibrinogen concentrate (Haemocomplettan ${ }^{\mathrm{TM}}$, CSL Behring, Marburg, Germany), prothrombin-complex concentrate (Beriplex ${ }^{\mathrm{TM}}$, CSL Behring) and blood products based on the results of Rotational Thrombelastometry (ROTEM ${ }^{\mathrm{TM}}$, Tem International $\mathrm{GmbH}$, Munich, Germany), multiple electrode aggregometry (Multiplate ${ }^{\mathrm{TM}}$, Roche Diagnostics International Ltd, Rotkreuz, Switzerland), and the 
static coagulation profile of the patients. The transfusion trigger was adjusted to the latest recommendations related to cardiac surgery. ${ }^{20,21}$

The immunosuppression therapy of the heart transplant recipients consisted of mycophenolate mofetil orally 90 minutes before the operation (1.5 g) and daily after the heart transplantation (2.0-3.0 g/ day); methylprednisolone $500 \mathrm{mg}$ IV prior to induction of anesthesia, $500 \mathrm{mg}$ IV 30 minutes after the aortic declamp, $125 \mathrm{mg}$ IV over the first 3 days, and then was continued as oral maintenance treatment; anti-thymocyte globulin IV infusion $(1.5 \mathrm{mg} / \mathrm{kg}$ ) for the first three postoperative days; tacrolimus orally $\left(0.1-0.2 \mathrm{mg}^{*} \mathrm{~kg}^{-1 *} \mathrm{day}^{-1}\right)$ from the fifth post-transplant day targeting serum concentration of 10-15 ng/ $\mathrm{mL}$. To exclude early graft rejection, patients were followed up with endomyocardial biopsy weekly during the first month after the heart transplantation. ${ }^{22}$

\subsection{Cytokine adsorption treatment}

Selection of heart transplant recipients to receive intraoperative cytokine adsorption (CA) treatment (CytoSorb ${ }^{\text {TM }}$, CytoSorbents Europe $\mathrm{GmbH}$, Berlin, Germany) was based on expert opinion. Endstage heart failure patients complicated by one or more organ dysfunctions prior to heart transplantation, poor functional status with expectation of developing severe VS in the perioperative period had priority for introducing intraoperative CytoSorb ${ }^{\mathrm{TM}}$ treatment. CytoSorb $^{\text {TM }}$ was applied for a one-cycle treatment during CPB. The 300-mL CytoSorb ${ }^{\mathrm{TM}}$ cartridge was positioned after the oxygenator providing near maximal blood flow through the cartridge via a specified side branch of the CPB circuit. The estimated flow rate through CytoSorb $^{\mathrm{TM}}$ was between $400 \mathrm{~mL} / \mathrm{min}$ and $500 \mathrm{~mL} / \mathrm{min}$ depending on the individual flow rate of CPB for all treatments. Taking into account the adsorption spectrum of CytoSorb ${ }^{\mathrm{TM}}$ (5-55 kDA fat soluble agents) and its possible effect on drug serum concentration, ${ }^{23}$ we considered methylprednisolone (molecular weight $<1 \mathrm{kDA}$, water soluble) as an immunosuppressive agent, which is not affected and mycophenolate-mofetil (MMF; molecular weight $<1 \mathrm{kDA}$, slightly water soluble: $\mathrm{pKa}=8.5$ ) as an immunosuppressive agent, which is partly, but not significantly affected by CytoSorb ${ }^{\mathrm{TM}}$ adsorption. Supplementary doses of MMF were not administered during the CPB period.

\section{5 | Measurement of inflammatory markers}

C-reactive protein (CRP) and procalcitonin (PCT) were measured from venous blood routinely at six time points during the perioperative period in all patients according to the institutional $\mathrm{OHT}$ protocol: prior to heart transplantation and daily for the first 5 days postoperatively. PCT was quantified by electrochemiluminescence immunoassay (Elecsys BRAHMS PCT, Roche Diagnostics GmbH, Mannheim, Germany), and CRP concentration was measured by particle enhanced turbidimetric assay (COBAS INTEGRA C-Reactive Protein Latex, Roche Diagnostics $\mathrm{GmbH}$ ). A PCT of $0.5 \mu \mathrm{g} / \mathrm{L}$ and CRP of $5.0 \mathrm{mg} / \mathrm{L}$ were considered as the upper limits of their normal ranges.

\section{6 | Outcome parameters}

Primary outcome was defined as hemodynamic stability and vasopressor demand during the first 48 hours postoperatively and magnitude of postoperative inflammatory response described by the kinetics of PCT and CRP. Secondary outcome parameters were the following: volume of postoperative bleeding; rate of reoperation for bleeding/ tamponade; need for blood products in the first 24 hours, length of $\mathrm{MV}$; rate of primary graft failure, postoperative mechanical circulatory support, acute kidney injury (AKI), postoperative renal replacement therapy (RRT), sepsis and early cardiac allograft rejection; length-ofICU stay, length-of-hospital stay, and 30-day mortality.

\section{7 | Statistical analysis}

Continuous variables were analyzed by the Shapiro-Wilk test for normality. Descriptive statistics of data were expressed as mean \pm standard deviation and median [interquartile range]. Categorical variables were presented as number of patients and percentage. Unpaired $t$ test, Mann-Whitney $U$ test, and $\chi^{2}$ test or Fisher's exact test were used for the unmatched comparisons of continuous and categorical variables where appropriate. To improve the imbalance in patients' baseline characteristics, we performed propensity score matching (PSM) using 1:1 match, nearest neighbor method with a caliper width of 0.2. ${ }^{24}$ After the univariate analyses of the parameters listed in Table 1 , variables that showed significant differences between the two groups were used as covariates in the PSM apart from Seattle Heart Failure Score, IMPACT score and high urgent status. Angiotensin converting enzyme inhibitor use, hemoglobin, bilirubin, C-reactive protein, procalcitonin, pretransplant hospitalization, and pretransplant inotrope support from the preoperative variables, and the number of packed red cell transfusions from the intraoperative variables were finally adjusted as covariates. The change in overall imbalance after PSM was analyzed for improvement calculating $L 1$ value and standardized mean differences of all covariates. ${ }^{25,26}$ The comparative analyses of outcome parameters in the matched cohort of control and treated patients were performed by paired $t$ test and Wilcoxon rank-sum test for continuous variables where appropriate and the McNemar test for categorical variables. To identify independent predictors of secondary outcome parameters, we used binomial logistic regression with step forward likelihood ratio method. Statistical significance was defined at $P<.05$ level in all tests. Analyses were performed with IBM SPSS Statistics for Windows, version 20.0. (IBM Corp., Armonk, NY, USA) and $R$-statistics for Windows, version 2.12.2 (R Foundation for Statistical Computing, Vienna, Austria).

\section{3 | RESULTS}

Ninety-one orthotopic heart transplantations were performed between January 2015 and December 2016 at the Heart and Vascular Centre of Semmelweis University. Seven patients were excluded from the study, of which five patients reached the exclusion criteria due to 
TABLE 1 Patient characteristics and perioperative clinical data in control and cytokine adsorption-treated groups

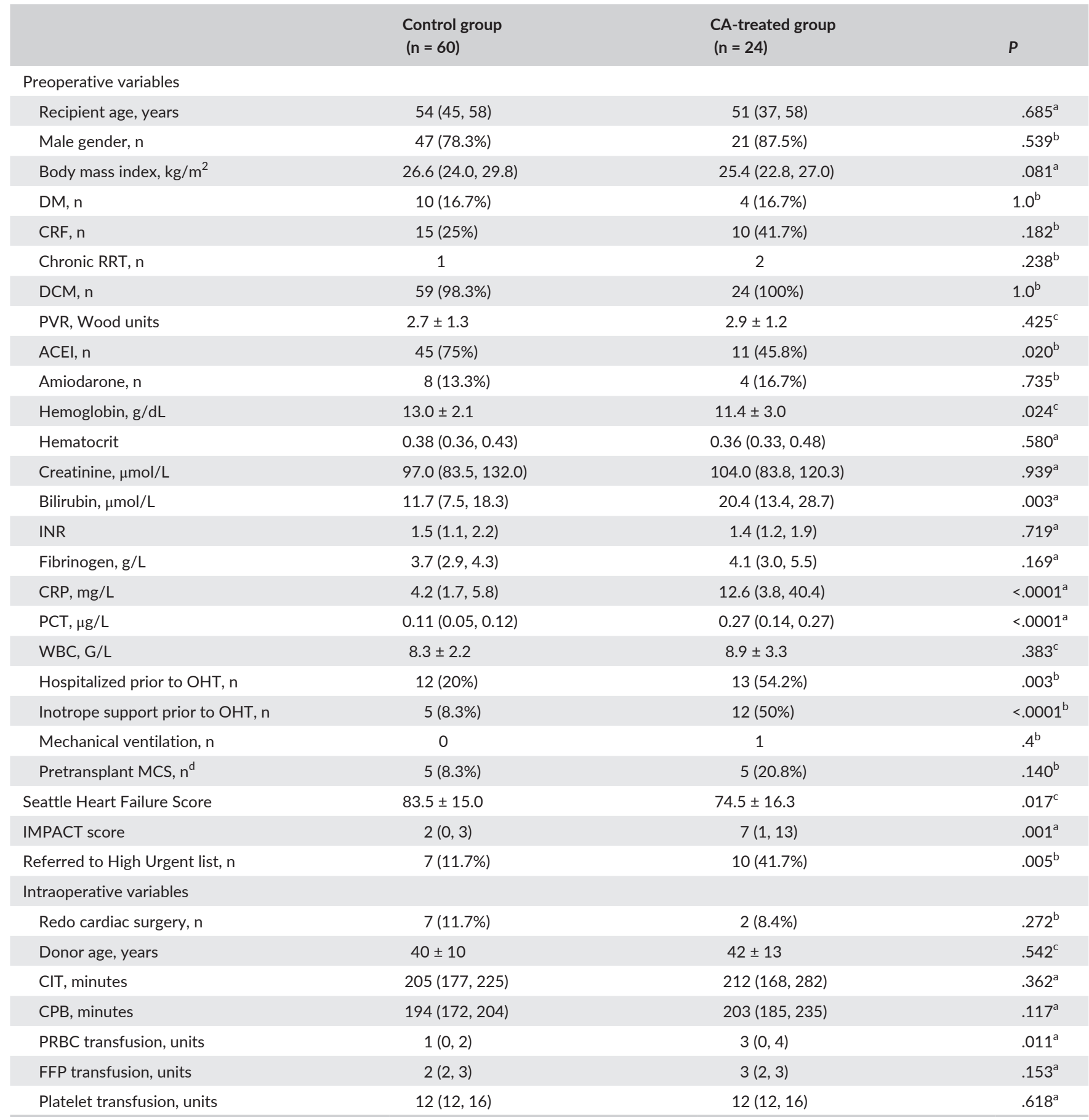

Data are expressed as median (interquartile range), number of patients (percent), and mean \pm standard deviation.

CA, cytokine adsorption; DM, diabetes mellitus; CRF, chronic renal failure; RRT, renal replacement therapy; DCM, dilatative cardiomyopathy; PVR, pulmonary vascular resistance; ACEI, angiotensin-converting enzyme inhibitor; INR, international normalized ratio; CRP, C-reactive protein; PCT, procalcitonin; WBC, white blood cell; OHT, orthotopic heart transplantation; MCS, mechanical circulatory support; IMPACT score, Index for Mortality Prediction After Cardiac Transplantation ${ }^{27}$; CIT, cold ischemic time; CPB, cardiopulmonary bypass; PRBC, packed red blood cell; FFP, fresh frozen plasma.

${ }^{a}$ Mann-Whitney $U$ test.

${ }^{\mathrm{b}} \chi^{2}$ test or Fisher's exact test.

'Unpaired $t$ test.

${ }^{\mathrm{d}}$ MCS consists of five left ventricular assist devices (LVAD) in the control group and three LVADs and two biventricular assist devices in the CA-treated group. 
TABLE 2 Propensity score-matched data of patient characteristics and perioperative clinical variables

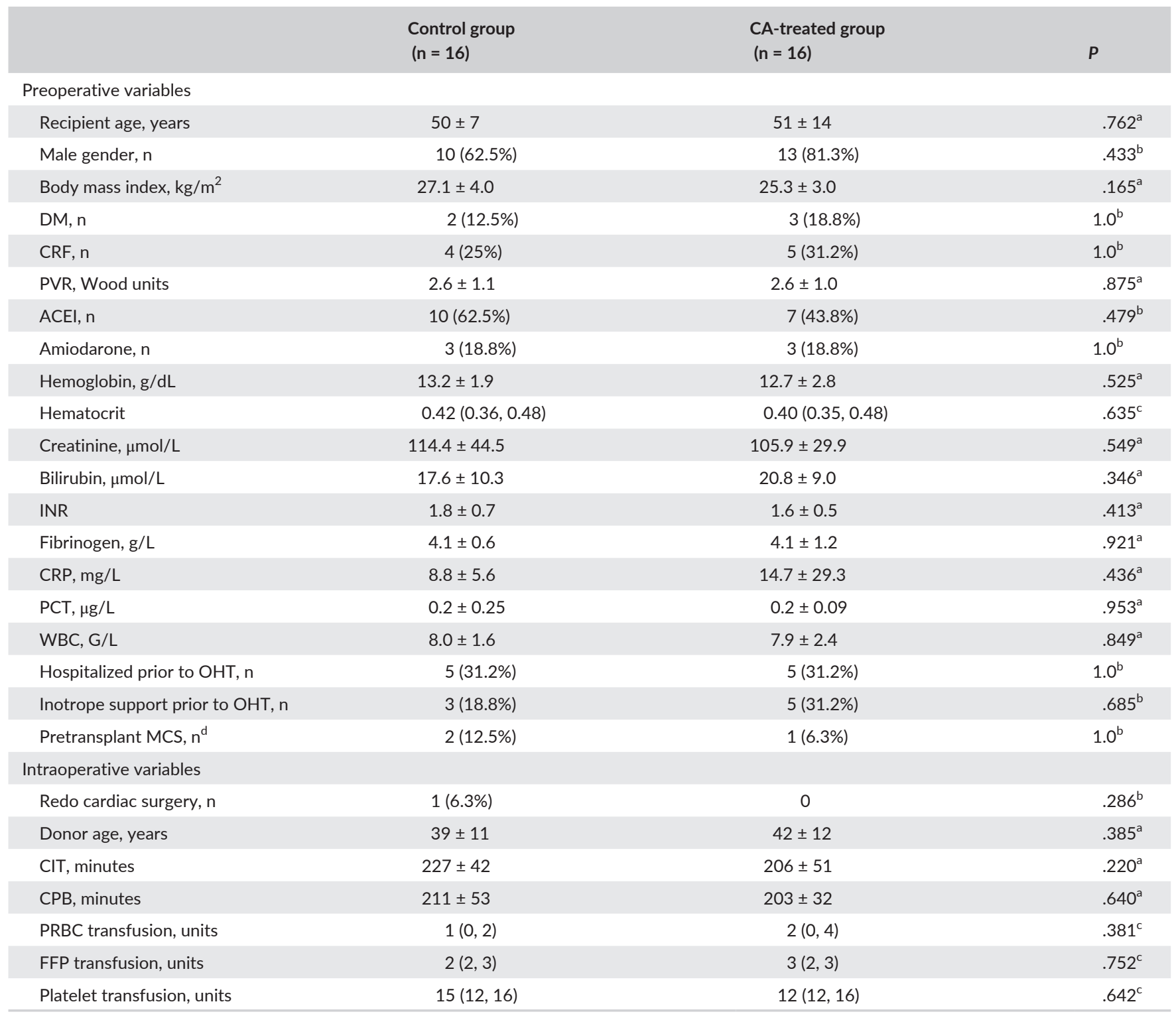

Data are presented as mean \pm standard deviation, number of patients (percent), and median (interquartile range).

The etiology of end-stage heart failure was dilatative cardiomyopathy in all cases of matched cohort.

CA, cytokine adsorption; DM, diabetes mellitus; CRF, chronic renal failure; PVR, pulmonary vascular resistance; ACEl, angiotensin-converting enzyme inhibitor; INR, international normalized ratio; CRP, C-reactive protein; PCT, procalcitonin; WBC, white blood cell; OHT, orthotopic heart transplantation; MCS, mechanical circulatory support; CIT, cold ischemic time; CPB, cardiopulmonary bypass; PRBC, packed red blood cell; FFP, fresh frozen plasma.

${ }^{a}$ Paired $t$ test.

${ }^{\mathrm{b}} \mathrm{McN}$ emar test.

${ }^{c}$ Wilcoxon rank-sum test.

${ }^{\mathrm{d}} \mathrm{MCS}$ consists of two left ventricular assist devices in the control group and one biventricular assist device in the CA-treated group.

death within 72 hours after the heart transplantation and other two patients had retransplantations. The mean age of the study population was $50 \pm 10$ years and $81 \%$ of them were male. Intraoperative CA was used in 24 cases (28.6\%). The baseline characteristics and the perioperative clinical data of the study cohort are shown in Table 1.

Propensity score matching from the study cohort of 60 controls and 24 CA-treated patients gave 16 matched pairs. After the matching $L_{1}$ decreased from 0.842 to 0.800 indicating an improvement in overall balance of covariates, and the absolute values of standardized mean differences were less than 0.25 in all baseline variables. Data of the matched population are summarized in Table 2 .

\section{1 | Primary outcome of propensity- matched comparison}

Median noradrenaline requirements were similar in the control and CA-treated groups during the heart transplantation procedure 0.15 $[0.05,0.22] \mu \mathrm{g}^{*} \mathrm{~kg}^{-1 *} \mathrm{~min}^{-1}$ vs $0.1[0.04,0.25] \mu \mathrm{g}^{*} \mathrm{~kg}^{-1 *} \mathrm{~min}^{-1}, P=.96$, 


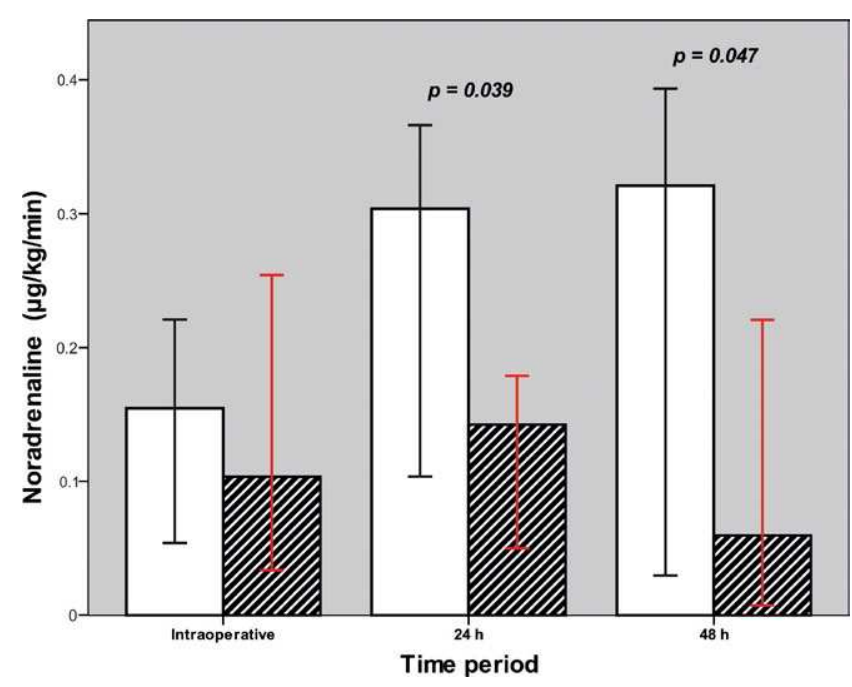

FIGURE 1 Comparison of noradrenaline requirements in the early postoperative period. Blank bar demonstrates control group and striped bar indicates CytoSorb ${ }^{\mathrm{TM}}$-treated patients. Data are presented as medians. Error bars show 95\% confidence interval. Wilcoxon ranksum test

respectively). However, it was significantly higher in the control group compared to the CA-treated group during the first 24 hours' posttransplantation $\left(0.3[0.12,0.35] \mu \mathrm{g}^{*} \mathrm{~kg}^{-1 *} \mathrm{~min}^{-1}\right.$ vs $0.14[0.05,0.18]$ $\mu \mathrm{g}^{*} \mathrm{~kg}^{-1 *} \min ^{-1}, P=.039$, respectively) and on the second postoperative day $\left(0.32[0.07,0.38] \mu \mathrm{g}^{*} \mathrm{~kg}^{-1 *} \mathrm{~min}^{-1} \mathrm{vs} 0.06[0.01,0.2] \mu \mathrm{g}^{*} \mathrm{~kg}^{-1 *} \mathrm{~min}^{-1}\right.$, $P=.047$, respectively) (Figure 1 ). The median terlipressin dose administered intraoperatively (control group: $0[0,0.24] \mu \mathrm{g}^{*} \mathrm{~kg}^{-1 *} \mathrm{~h}^{-1} \mathrm{vs}$ CA-treated group: $\left.0[0,0.3] \mu \mathrm{g}^{*} \mathrm{~kg}^{-1 *} \mathrm{~h}^{-1}, P=.94\right)$ and on the first posttransplant day (control group: $0.7[0.05,0.8] \mu \mathrm{g}^{*} \mathrm{~kg}^{-1 *} \mathrm{~h}^{-1}$ vs CA-treated group: $0[0,0.6] \mu \mathrm{g}^{*} \mathrm{~kg}^{-1 *} \mathrm{~h}^{-1}, P=.16$ ) were similar in both groups. Nevertheless, the terlipressin requirement was less in CA-treated patients than in controls on the second postoperative day with marginal significance $\left(0[0,0.4] \mu \mathrm{g}^{*} \mathrm{~kg}^{-1 *} \mathrm{~h}^{-1}\right.$ vs $0.7[0.05,1.2] \mu \mathrm{g}^{*} \mathrm{~kg}^{-1 *} \mathrm{~h}^{-1}$, $P=.06$, respectively). The median duration of vasopressor (controls: 5 $[2,11]$ days vs CA-treated patients: $4[2,5]$ days, $P=1.0$ ) as well as inotrope therapy (controls: $12[8,19]$ days vs CA-treated patients: $8[6,9]$ days, $P=.80$ ) was equal in both groups. Primary hemodynamic parameters did not show any differences between the control and CA-treated groups as demonstrated by the average $\mathrm{Cl}\left(3.4 \pm 1.1 \mathrm{~L}^{*} \mathrm{~min}^{-1 *} \mathrm{~m}^{-2}\right.$ vs $3.2 \pm 0.9 \mathrm{~L}^{*} \mathrm{~min}^{-1 *} \mathrm{~m}^{-2}, P=.29$, respectively), MAP $(71 \pm 8 \mathrm{~mm} \mathrm{Hg}$ vs $74 \pm 8 \mathrm{~mm} \mathrm{Hg}, P=.12$, respectively), systemic vascular resistance index (SVRI; $1480 \pm 499$ dyne* $\mathrm{s}^{*} \mathrm{~cm}^{-5 *} \mathrm{~m}^{-2}$ vs $1495 \pm 443$ dyne* $\mathrm{s}^{*} \mathrm{~cm}^{-5 *} \mathrm{~m}^{-2}$, $P=.89$, respectively), and PVR $(1.7 \pm 1.0$ Wood unit vs $2.0 \pm 0.8$ Wood unit, $P=.42$, respectively) during the first 24 hours postoperatively. $\mathrm{SvO}_{2}$ was in the normal range and similar in both groups (control group: $71.2 \pm 9.7 \%$ vs CA-treated group: $71.2 \pm 7.7 \%, P=1.0$ ). The results of the hemodynamic parameters and oxygenation measured at the five time points in the first 24 hours can be seen in Figures 2-4. The time courses of lactate concentration were divergent with an overall lower concentration in the CA-treated group compared to the control group but the differences did not reach the level of significance at the measurement points (Figure 4, panel B).
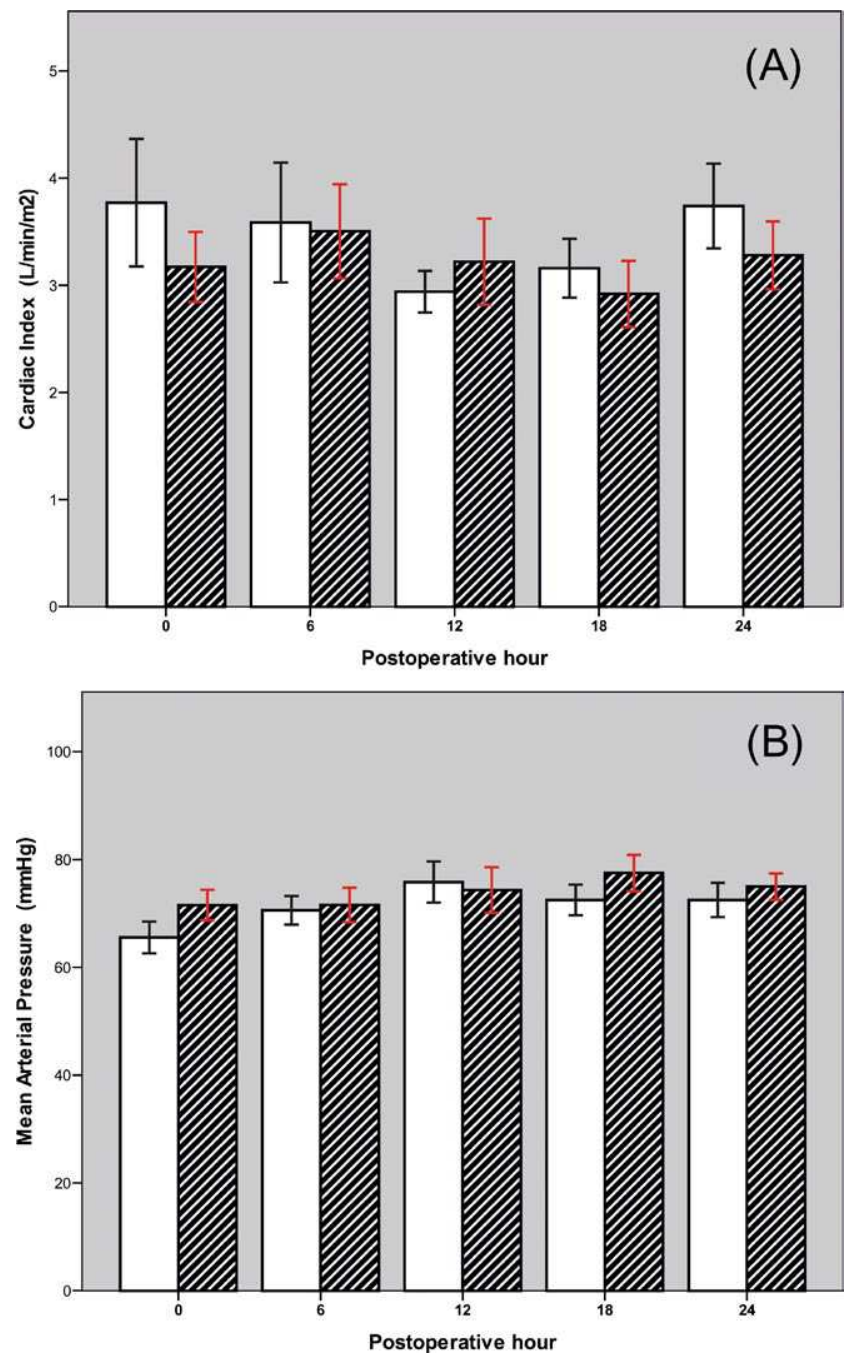

FIGURE 2 Cardiac index (A) and mean arterial pressure (B) during the first $24 \mathrm{~h}$ after orthotopic heart transplantation. Blank bar demonstrates controls and striped bar indicates CytoSorb ${ }^{\mathrm{TM}}$-treated patients. Data are presented as means. Error bars show standard error of the mean. Paired $t$ test

Procalcitonin and CRP changes showed pronounced postoperative inflammatory responses in the two groups. PCT peaked on the first postoperative day and then had a tendency to decrease over subsequent days (Figure 5, panel A). CRP, however, reached its highest levels on the third postoperative day and declined after that (Figure 5, panel B). The dynamics of PCT and CRP did not vary between the control- and CA-treated groups. Interestingly, while the PCT peak showed a strong correlation with noradrenaline requirements in the first 24 hours in the control group $\left(r^{2}=.63, P=.006\right)$, there was no correlation in the CA-treated group $\left(r^{2}=.06, P=.51\right)$.

\section{2 | Secondary outcome of propensity- matched comparison}

There were no differences in postoperative blood loss and number of transfused blood products (ie, packed red blood cell, fresh frozen 

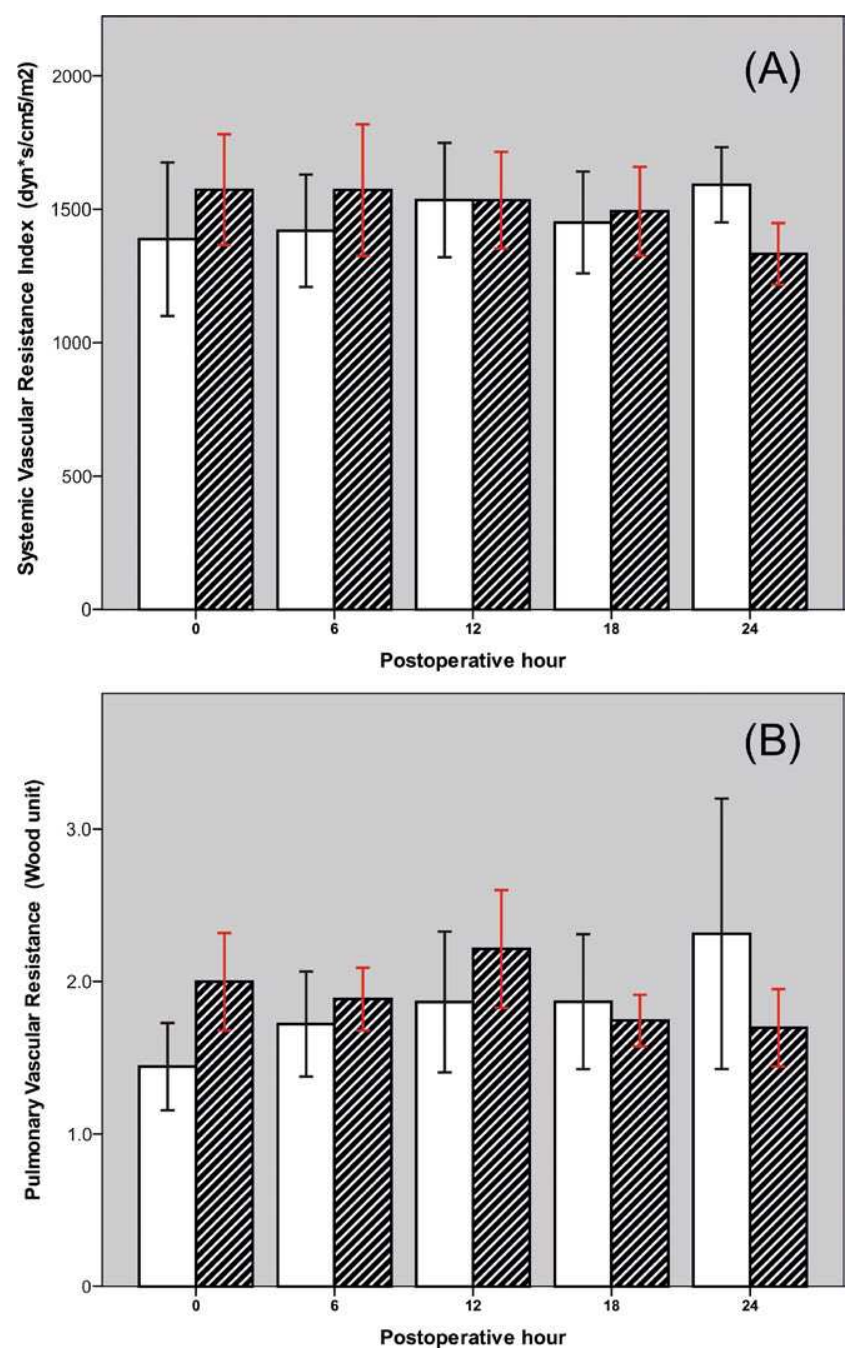

FIGURE 3 Trends of systemic vascular resistance (A) and pulmonary vascular resistance (B) over the first $24 \mathrm{~h}$ postoperatively in cytokine adsorption treated patients (striped bar) and control group (blank bar). Data are demonstrated as means. Error bars correspond to standard error of the mean. Paired $t$ test

plasma, and platelets) between the two groups (Table 3). Need for re-exploration for bleeding or tamponade was less in the CA-treated patients than in the control group with marginal significance (2 vs 5 cases, $P=.06$, respectively). Patients in the CA-treated group experienced shorter ventilation times and less acute kidney injury but the differences were not statistically significant. Nevertheless, the frequency of acute or acute-on-chronic renal failure requiring renal replacement therapy was higher in the control group than in CA-treated group ( 4 vs 2 cases, $P=.031$, respectively). The incidence of sepsis and early graft rejection did not vary in the two groups. While we did not find any difference in length-of-hospital stay between the two groups, the length-of-ICU stay was shorter in CA-treated group than in control group (10 vs 20 days, respectively) but this difference was not statistically significant. There were no deaths during the first month after transplantation in CA-treated patients compared to two cases in the propensity-matched control group. Details of postoperative complications are summarized in Table 3. The independent predictors of
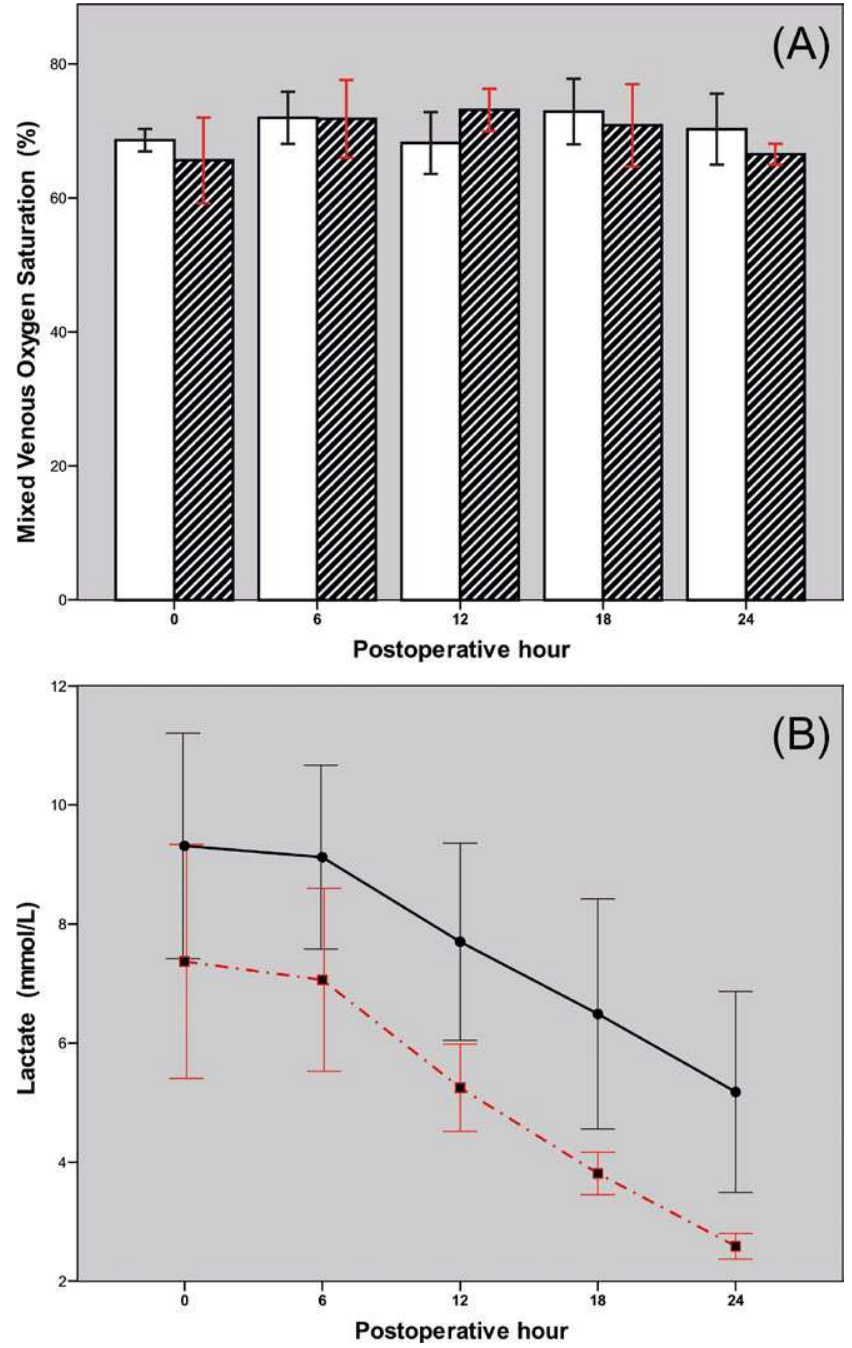

FIGURE 4 Postoperative changes in mixed venous oxygen saturation (A) and lactate level (B) in the early postoperative period. While mixed venous oxygen saturation did not differ between CytoSorb $^{\mathrm{TM}}$-treated patients (striped bar) and controls (blank bar), a substantial difference was seen in the time courses of lactate of the two groups. The lactate concentration normalized faster, within $24 \mathrm{~h}$, in the CytoSorb ${ }^{\text {TM }}$ treated group (red dotted line) than the control group (black continuous line). Data are demonstrated as means. Error bars correspond to standard error of the mean. Paired $t$ test

secondary outcome parameters analyzed in the nonmatched cohort are shown in Table 4.

\section{4 | DISCUSSION}

This propensity score-matched cohort study aimed to explore the influence of intraoperative use of CytoSorb ${ }^{\mathrm{TM}}$ treatment on postoperative vasoplegia, inflammatory response, and clinical outcome in patients undergoing OHT. Our study showed that CA treatment was associated with less severe postoperative vasoplegia and reduced vasopressor demand in the first 48 hours postoperatively, despite similar kinetics of the inflammatory markers PCT and CRP in the 

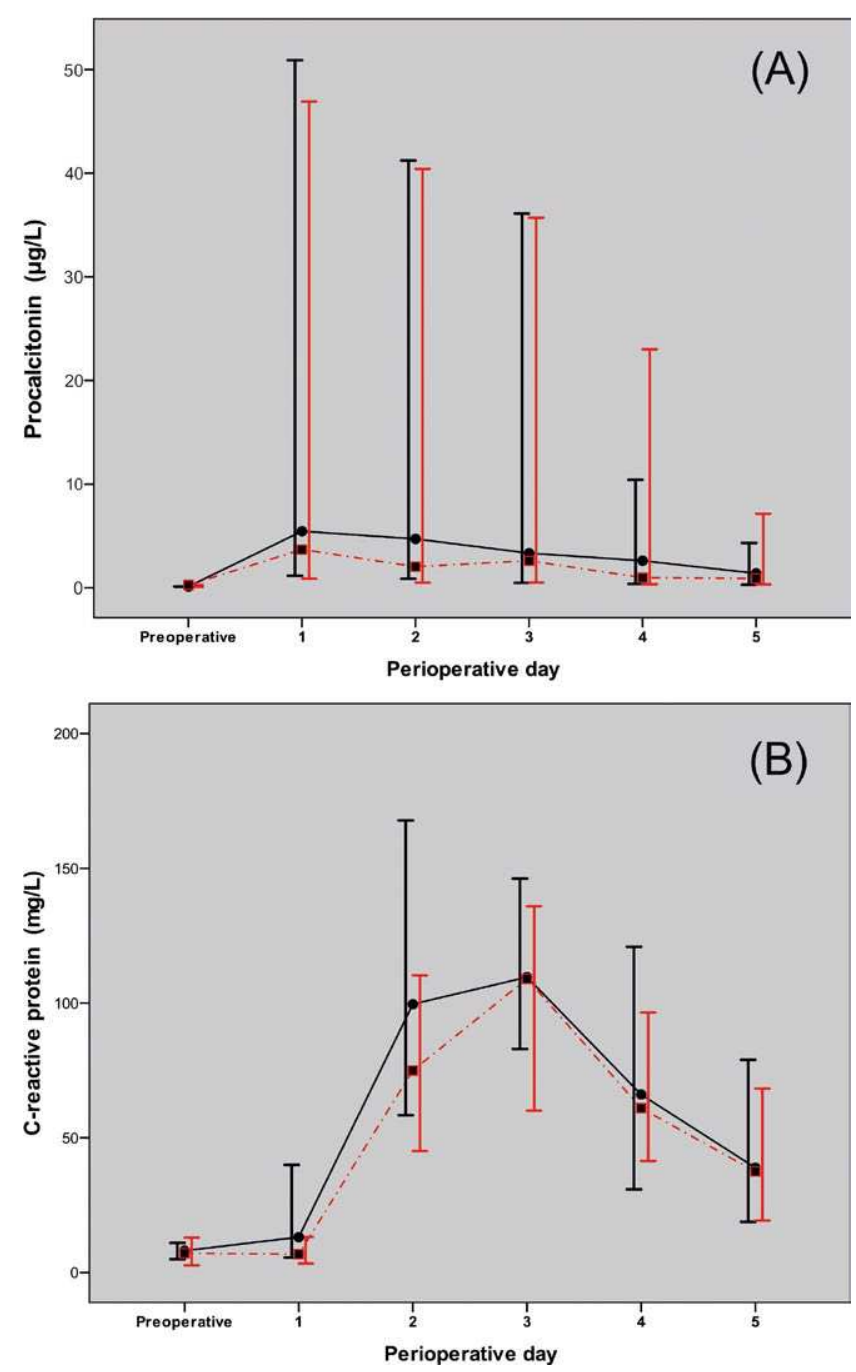

FIGURE 5 Comparison of postoperative inflammatory responses demonstrated by dynamics of procalcitonin (A) and C-reactive protein (B). Black continuous line demonstrates control group and red dotted line indicates CytoSorb ${ }^{\text {TM }}$ treated group. Data are presented as medians. Error bars correspond to $95 \%$ confidence interval. Wilcoxon rank-sum test

two groups. Patients treated with CytoSorb ${ }^{\mathrm{TM}}$ experienced shorter periods of postoperative MV and ICU stay. In fact, we observed less $\mathrm{AKI}$ and RRT requirement in CA-treated group than in the controls. Additionally, this study did not confirm higher rates of complications including postoperative bleeding, pericardial tamponade, sepsis, and early graft rejection in the CA-treated group.

Patients with end-stage heart failure undergoing $\mathrm{OHT}$ are at a remarkably higher risk for developing severe vasoplegia compared to nontransplant cardiac surgery patients. ${ }^{4,5,28}$ One of the main contributing factors of this difference is the post-CPB inflammatory response. This is more pronounced in OHT than in conventional cardiac surgery patients due to the pre-existing activation of the immune system by the advanced heart failure and reaction of the host immune system to graft tissue. ${ }^{4,29}$ The rational to introduce the pre-emptive intraoperative CytoSorb ${ }^{\mathrm{TM}}$ treatment among OHT recipients was to mitigate and modify this aggravated post-CPB inflammatory response and hopefully prevent development of severe vasoplegia with excessive vasopressor demand.

Case reports and a case series published recently demonstrated that CytoSorb ${ }^{\text {TM }}$ treatment of patients with septic shock results in improved hemodynamics, significant decrease in vasopressor dose, and effective removal of pro-inflammatory cytokines after 24 hours of application. ${ }^{16,17,19,30}$ Träger et al confirmed the beneficial effects of postoperative CytoSorb ${ }^{\mathrm{TM}}$ treatment on hemodynamic stability and the control of elevated pro-inflammatory cytokines in cases of severe post-CPB SIRS complicated with AKI in a retrospective analysis of 16 patients. ${ }^{18}$ However, Bernardi et al found no significant differences in pro-inflammatory cytokine levels and clinical outcome in cardiac surgical patients when they used CytoSorb ${ }^{\text {TM }}$ treatment intraoperatively in a blinded, randomized, controlled pilot study. ${ }^{31}$ They highlighted the length of treatment time (191 \pm 56 minutes), concentrationdependent adsorption of cytokines, and strong inter-individual differences in cytokine levels as possible reasons for their findings. ${ }^{31}$ Our study demonstrated significantly less vasopressor requirements in the CA-treated patients than in controls in the first 48 hours postoperatively (Figure 1) after an intraoperative mean treatment time of $203 \pm 32$ minutes. Despite the lower doses of noradrenaline and terlipressin administered in the CA-treated group in our study, we found similar parameters to those of controls for both hemodynamics (Figures 2-3) and tissue oxygenation (Figure 4A). However, lactate concentrations were lower in the CA-treated patients than in controls over the first postoperative 24 hours (Figure 4B). Additionally, confounding factors in the pre- and intraoperative periods, known to influence postoperative vasopressor demand, were well balanced in the propensity score-matched cohort (for details see Table 2). These data suggest that a shorter period of intraoperative CytoSorb ${ }^{\mathrm{TM}}$ treatment (ie, <4 hours) can be clinically effective for controlling postoperative vasoplegia in OHT patients.

We analyzed PCT and CRP as inflammatory markers in the first five postoperative days to quantify post-CPB inflammatory response. $\mathrm{CRP}$ has some limitations in the evaluation of post-CPB inflammatory response, especially in the early postoperative phase. CRP increases after cardiac surgery regardless of the magnitude of SIRS or surgical trauma. ${ }^{32,33}$ PCT is a widely used diagnostic and follow-up marker of infective or noninfective SIRS. ${ }^{32,34-36}$ Pro-inflammatory cytokines contribute to early PCT induction after surgery resulting in a peak concentration within 24 hours. $^{32,34}$ The peak level of PCT correlates well with the extent of surgical trauma, duration of aortic cross-clamp and CPB, and its dynamics can be a prognostic indicator of complications after cardiac surgery or $\mathrm{OHT} .^{32,34,35}$ In this study, we did not see differences in peak levels and kinetics of CRP and PCT between the CA-treated and control groups representing similar post-CPB inflammatory responses. However, we found a strong correlation between the PCT peak and the noradrenaline requirement measured on the first postoperative day in the control group, which was not observed in the CA-treated patients. This interesting result is indicative of a possible influence of CytoSorb ${ }^{\mathrm{TM}}$ treatment on vasoactive mediators associated with $\mathrm{CPB}$ and $\mathrm{OHT}$. 
TAB LE 3 Comparison of clinical outcome parameters in propensity score matched data

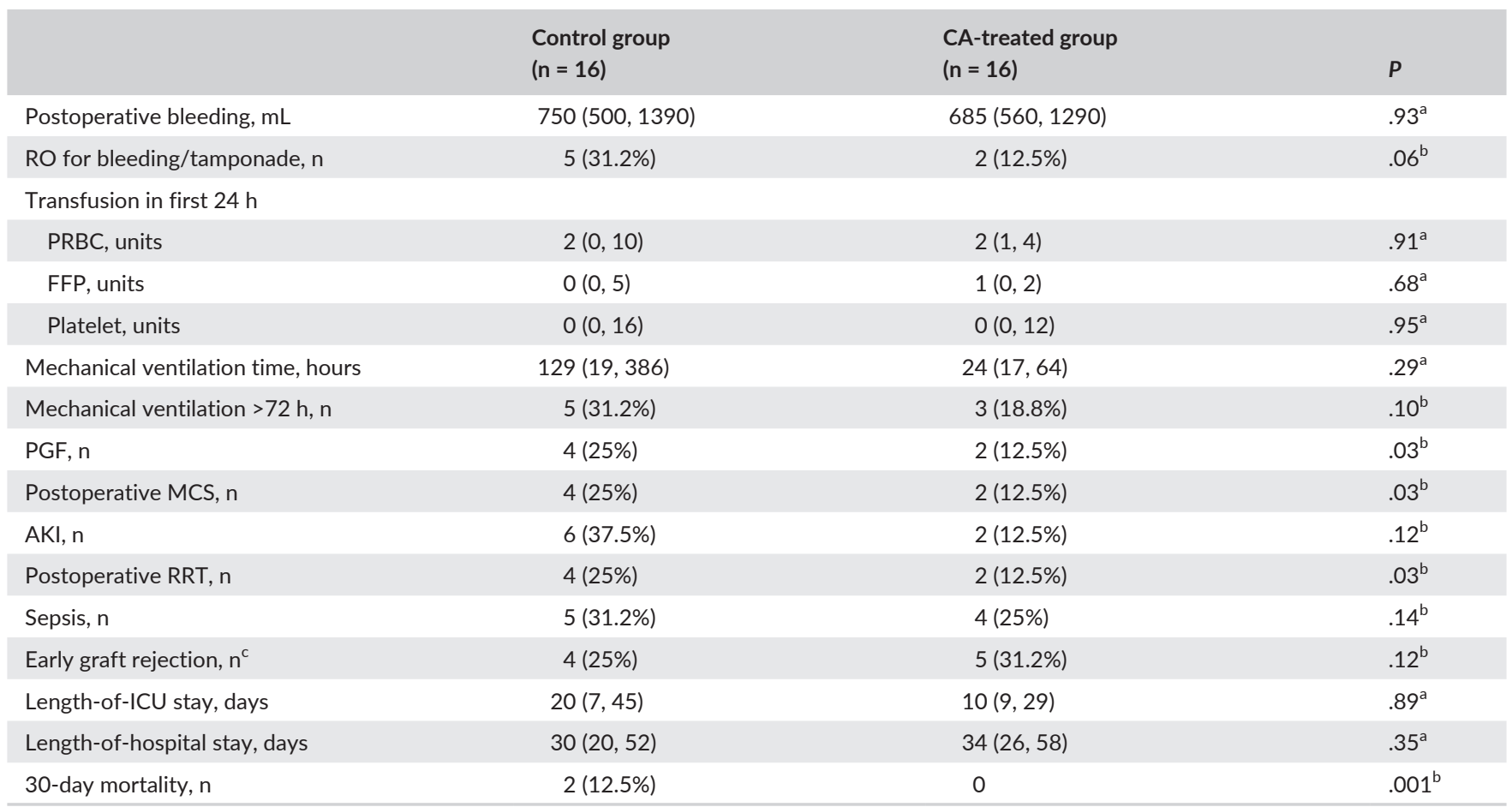

Data are presented as median (interquartile range), number of patients (percent).

CA, cytokine adsorption; RO, reoperation; PRBC, packed red blood cell; FFP, fresh frozen plasma; PGF, primary graft failure; MCS, mechanical circulatory support; AKI, acute kidney injury; RRT, renal replacement therapy; ICU, intensive care unit.

${ }^{a}$ Wilcoxon rank-sum test.

${ }^{\mathrm{b}} \mathrm{McNemar}$ test.

${ }^{c}$ Early graft rejection was defined by the first surveillance endomyocardial biopsy (EMB) performed on the seventh post-transplant day. EMB grade (International Society for Heart and Lung Transplantation) $\geq 1 \mathrm{~A}$ was considered as a positive result for early graft rejection. Based on the first 12 months follow-up data, the frequency of the cardiac allograft vasculopathy was four cases in the CA-treated group vs 0 cases in the control group, $P<.0001$.

Peng and co-workers confirmed intense cytokine removal, better hemodynamics, and short-term survival after 3 hours of hemoadsorption treatment compared to sham therapy in an animal model of

TABLE 4 Independent predictors of secondary outcome parameters

\begin{tabular}{|c|c|c|c|}
\hline & OR & $95 \% \mathrm{Cl}$ & $P$ \\
\hline \multicolumn{4}{|l|}{ Postoperative bleeding: } \\
\hline Postoperative MCS support & 4.7 & $1.2-18.9$ & .031 \\
\hline \multicolumn{4}{|l|}{ Mechanical ventilation $>72 \mathrm{~h}$ : } \\
\hline AKI & 6.7 & $1.9-23.8$ & .003 \\
\hline $\begin{array}{l}\text { Intraoperative PRBC } \\
\text { transfusion }>4 \text { units }\end{array}$ & 11.6 & $2.0-67.7$ & .007 \\
\hline \multicolumn{4}{|l|}{ Acute kidney injury: } \\
\hline Postoperative MCS support & 5.6 & $1.5-20.8$ & .010 \\
\hline \multicolumn{4}{|l|}{ Sepsis: } \\
\hline Hospitalization prior to $\mathrm{OHT}$ & 3.3 & $1.2-9.2$ & .024 \\
\hline
\end{tabular}

Binomial logistic regression, step forward likelihood ratio.

Logistic regression did not identify independent predictors of primary graft failure and early graft rejection.

$\mathrm{OR}$, odds ratio; $\mathrm{Cl}$, confidence interval; $\mathrm{MCS}$, mechanical circulatory support; AKI, acute kidney injury; PRBC, packed red blood cell; OHT, orthotopic heart transplantation. sepsis. ${ }^{14}$ Kogelmann et al reported lower mortality than predicted by the APACHE II score associated with early CytoSorb $^{\text {TM }}$ treatment in patients with septic shock. ${ }^{19}$ Interestingly, these favorable effects of hemoadsorption on outcome were not completely reproducible in the cardiac surgery setting by recent investigations. ${ }^{18,31}$ In our study, clinical outcome showed a positive tendency in the CA-treated patients compared to controls especially in length of MV, ICU stay, frequency of RRT, and mortality although the observed differences in these parameters did reach the level of statistical significance, due, in part, to the small sample size. Furthermore, the complication profile of CA-treated patients was similar but definitely not inferior to controls in terms of postoperative bleeding, sepsis, and early graft rejection. These positive results can be linked at least in part to significantly less vasopressor demand in the early postoperative period associated with intraoperative CytoSorb ${ }^{\mathrm{TM}}$ treatment.

\section{1 | Limitations}

Our investigation does have limitations. This observational study was carried out in a single center which influenced the sample size for the study period. Patient selection for the use of intraoperative cytokine adsorption was based on the arbitrary criteria of clinical experts. This is due to the scarcity of published data in literature related to CytoSorb $^{\text {TM }}$ 
treatment, particularly in field of cardiac surgery or OHT due the novelty of this technology. This study completely adhered to the institutional protocol for OHT; therefore, the postoperative inflammatory response was described only by changes in PCT and CRP, and no other pro-inflammatory cytokines for more specific information. These limitations somewhat restrict the interpretation of our results.

\section{5 | CONCLUSIONS}

To the best of our knowledge, this is the first observational study presenting the use of intraoperative CytoSorb ${ }^{T M}$ treatment during $\mathrm{OHT}$. In this study, CytoSorb ${ }^{\mathrm{TM}}$ treatment was associated with significantly reduced vasopressor requirements and less frequent RRT in the early postoperative period. The favorable trends in length of $\mathrm{MV}$, ICU stay, and rate of AKI observed in the CA-treated patients suggest that intraoperative CytoSorb ${ }^{\mathrm{TM}}$ treatment might have benefit for reducing postoperative complications and improving outcome in the heart transplantation setting via more stable hemodynamics and reduced vasopressor demand. This study confirms that intraoperative cytokine adsorption is not linked to a higher rate of postoperative adverse events such as bleeding, sepsis, or early cardiac allograft rejection.

\section{CONFLICTS OF INTERESTS}

First author received travel funding from CytoSorbents Europe $\mathrm{GmbH}$, Berlin, Germany. All other authors declare that they have no competing financial or other interest in relation to their work.

\section{AUTHORS' CONTRIBUTIONS}

All authors have made substantial contributions to conception and design of the study. EN, EK, KR, AS, SS, NK, GC, KBK, VB, GT, and SF participated in acquisition, analysis, and interpretation of data. All authors have been involved in drafting and review of the manuscript. All authors read and approved the final manuscript.

\section{ORCID}

Endre Nemeth iD http://orcid.org/0000-0001-6033-4297

\section{REFERENCES}

1. Levin MA, Lin HM, Castillo JG, Adams DH, Reich DL, Fischer GW. Early on-cardiopulmonary bypass hypotension and other factors associated with vasoplegic syndrome. Circulation. 2009;120:1664-1671.

2. Omar S, Zedan A, Nugent K. Cardiac vasoplegia syndrome: pathophysiology, risk factors and treatment. Am J Med Sci. 2015;349:80-88.

3. Weis F, Kilger E, Beiras-Fernandez A, et al. Association between vasopressor dependence and early outcome in patients after cardiac surgery. Anaesthesia. 2006;61:938-942.

4. Byrne JG, Leacche M, Paul S, et al. Risk factors and outcomes for 'vasoplegia syndrome' following cardiac transplantation. Eur J Cardiothorac Surg. 2004;25:327-332.
5. Patarroyo M, Simbaqueba C, Shrestha K, et al. Pre-operative risk factors and clinical outcomes associated with vasoplegia in recipients of orthotopic heart transplantation in the contemporary era. J Heart Lung Transplant. 2012;31:282-287.

6. Shanmugam G. Vasoplegic syndrome - the role of methylene blue. Eur J Cardio-thorac Surg. 2005;28:705-710.

7. Hasper D, Hummel M, Kleber FX, Reindl I, Volk HD. Systemic inflammation in patients with heart failure. Eur Heart J. 1998;19:761-765.

8. Deswal A, Petersen NJ, Feldman AM, Young JB, White BG, Mann DL. Cytokines and cytokine receptors in advanced heart failure: an analysis of the cytokine database from the Vesnarinone Trial (VEST). Circulation. 2001;103:2055-2059.

9. Laffey JG, Boylan JF, Cheng DCH. The systemic inflammatory response to cardiac surgery. Anesthesiology. 2002;97:215-252.

10. Parolari A, Camera M, Alamanni F, et al. Systemic inflammation after on-pump and off-pump coronary bypass surgery: a one-month follow-up. Ann Thorac Surg. 2007;84:823-828.

11. Hosseinian L, Weiner M, Levin MA, Fischer GW. Methylene blue: magic bullet for vasoplegia? Anesth Analg. 2016;122:194-201.

12. Kellum JA, Song M, Venkataraman R. Hemoadsorption removes tumor necrosis factor, interleukin-6, and interleukin-10, reduces nuclear factor-кB DNA binding, and improves short-term survival in lethal endotoxemia. Crit Care Med. 2004;32:801-805.

13. Kellum JA, Venkataraman R, Powner D, Elder M, Hergenroeder G, Carter M. Feasibility study of cytokine removal by hemoadsorption in brain-dead humans. Crit Care Med. 2008;36:268-272.

14. Peng ZY, Carter MJ, Kellum JA. Effects of hemoadsorption on cytokine removal and short-term survival in septic rats. Crit Care Med. 2008;36:1573-1577.

15. Taniguchi T. Cytokine adsorbing columns. Contrib Nephrol. 2010;166:134-141.

16. Mitzner SR, Gloger M, Henschel J, Koball S. Improvement of hemodynamic and inflammatory parameters by combined hemoadsorption and hemodiafiltration in septic shock: a case report. Blood Purif. 2013;35:314-315.

17. Hetz H, Berger R, Recknagel P, Steltzer H. Septic shock secondary to $\beta$-hemolytic streptococcus-induced necrotizing fasciitis treated with a novel cytokine adsorption therapy. Int J Artif Organs. 2014;37:422-426.

18. Träger K, Fritzler D, Fischer G, et al. Treatment of post-cardiopulmonary bypass SIRS by hemoadsorption: a case series. Int J Artif Organs. 2016;39:141-146.

19. Kogelmann K, Jarczak D, Scheller M, Drüner M. Hemoadsorption by CytoSorb in septic patients: a case series. Crit Care. 2017;21:74.

20. Ferraris VA, Brown JR, Despotis GJ, et al. 2011 update to the society of thoracic surgeons and the society of cardiovascular anesthesiologists blood conservation clinical practice guidelines. Ann Thorac Surg. 2011;91:944-982.

21. Kozek-Langenecker SA, Afshari A, Albaladejo P, et al. Management of severe perioperative bleeding: guidelines from the European Society of Anaesthesiology. Eur J Anaesthesiol. 2013;30:270-382.

22. Costanzo MR, Dipchand A, Starling R, et al. The international society of heart and lung transplantation guidelines for the care of heart transplant recipients. J Heart Lung Transplant. 2010;29: 914-956.

23. Reiter K, Bordoni V, Dall'Olio G, et al. In vitro removal of therapeutic drugs with a novel adsorbent system. Blood Purif 2002;20: 380-388.

24. Lunt M. Selecting an appropriate caliper can be essential for achieving good balance with propensity score matching. Am J Epidemiol. 2014;179:226-235.

25. lacus SM, King G, Porro G. Software for coarsened exact matching. J Stat Softw. 2009;30:1-27.

26. Stuart EA. Matching methods for causal inference: a review and a look forward. Stat Sci. 2010;25:1-21. 
27. Weiss ES, Allen JG, Arnaoutakis GJ, et al. Creation of a quantitative recipient risk index for mortality prediction after cardiac transplantation (IMPACT). Ann Thorac Surg. 2011;92:914-922.

28. Carrel T, Englberger L, Mohacsi P, Neidhart P, Schmidli J. Low systemic vascular resistance after cardiopulmonary bypass: incidence, etiology, and clinical importance. J Card Surg. 2000;15:347-353.

29. Wan S, Marchant A, DeSmet JM, et al. Human cytokine responses to cardiac transplantation and coronary artery bypass grafting. J Thorac Cardiovasc Surg. 1996;111:469-477.

30. David S, Thamm K, Schmidt BMW, Falk CS, Kielstein JT. Effect of extracorporeal cytokine removal on vascular barrier function in a septic shock patient. J Intensive Care. 2017;5:12.

31. Bernardi MH, Rinoesl H, Dragosits K, et al. Effect of hemoadsorption during cardiopulmonary bypass surgery - a blinded, randomized, controlled pilot study using a novel adsorbent. Crit Care. 2016;20:96.

32. Sponholz C, Sakr Y, Reinhart K, Brunkhorst F. Diagnostic value and prognostic implications of serum procalcitonin after cardiac surgery: a systematic review of the literature. Crit Care. 2006;10:R145.

33. Delannoy B, Guye ML, Slaiman DH, Lehot JJ, Cannesson M. Effect of cardiopulmonary bypass on activated partial thromboplastin time waveform analysis, serum procalcitonin and C-reactive protein concentrations. Crit Care. 2009;13:R180.

34. Meisner M, Rauschmayer C, Schmidt J, et al. Early increase of procalcitonin after cardiovascular surgery in patients with postoperative complications. Intensive Care Med. 2002;28:1094-1102.

35. Amin DN, Pruit JC, Schuetz P. Influence of major cardiopulmonary surgery on serum levels of procalcitonin and other inflammatory markers. Anaesth Intensive Care. 2012;40:760-766.

36. Meisner M. Update on procalcitonin measurements. Ann Lab Med. 2014;34:263-273.

How to cite this article: Nemeth E, Kovacs E, Racz K, et al. Impact of intraoperative cytokine adsorption on outcome of patients undergoing orthotopic heart transplantation-an observational study. Clin Transplant. 2018;32:e13211.

https://doi.org/10.1111/ctr.13211 